\title{
Avaliação laboratorial de misturas asfálticas à quentes produzidas com grafite
}

\section{cominuído}

\author{
Laboratory evaluation of hot asphalt mixtures produced with combined graphite \\ Evaluación de laboratorio de mezclas asfálticas calientes producidas con grafito triturado
}

Recebido: 16/01/2021 | Revisado: 19/01/2021 | Aceito: 07/02/2021 | Publicado: 14/02/2021

\author{
Alex Gomes Pereira \\ ORCID: https://orcid.org/0000-0003-3563-4161 \\ Centro Universitário São Lucas, Brasil \\ E-mail: alexgp885@gmail.com \\ Cristiano da Silva Vieira \\ ORCID: https://orcid.org/0000-0002-1276-0336 \\ Centro Universitário São Lucas, Brasil \\ E-mail: cristiano.vieira@saolucas.edu.br \\ Marcelo Batista de Oliveira \\ ORCID: https://orcid.org/0000-0003-4921-968X \\ Universidade Federal de Rondônia, Brasil \\ E-mail: marcelo@unir.br \\ Júnior Cleber Alves Paiva \\ ORCID: https://orcid.org/0000-0002-6938-4351 \\ Faculdade de Ciências e de Tecnologia de Rondônia, Brasil \\ E-mail: juniorcleber.ro@gmail.com \\ Rafael Luis da Silva \\ ORCID: https://orcid.org/0000-0002-9485-479X \\ Centro Universitário São Lucas, Brasil \\ E-mail: rafaeluismat@gmail.com
}

\begin{abstract}
Resumo
Ao longo de sua história, a construção de pavimentos rodoviários, enfrenta a perda das características funcionais e estruturais, em razão dos obstáculos tecnológicos e financeiros. Dessa forma, tornam-se relevantes os estudos de novos materiais a serem utilizados em concretos asfálticos, a fim de favorecer a implementação de políticas de manutenção, recuperação e projetos de pavimentos mais econômicos pela sua maior adequabilidade técnica a longo prazo, e, em especial, minimizar a manutenção precoce dessas estruturas. Neste aspecto, o objetivo principal do presente trabalho foi compara experimentalmente por meio módulo complexo $\left(\mathrm{E}^{*}\right)$ e ângulo de fase $(\delta)$ dois tipos de concreto asfáltico, uma confeccionada com composição asfáltica com cimento Portland (CA-REF), e a outra composição asfáltica com grafite cominuído (CA-GRAFC). Para isto, empregou-se o equipamento de flexão a quatro pontos, sendo utilizado um carregamento sinusoidal uniaxial de compressão, amplitude de deformação de $50 \mu \mathrm{m} / \mathrm{m}$; frequências de $0,1,0,2,0,5,1,2,5,10$ e $20 \mathrm{~Hz}$ e temperaturas de 0 a $40^{\circ} \mathrm{C}$, com incrementos de $5^{\circ} \mathrm{C}$. Após a realização dos ensaios mecânicos, constatou-se que, sob as condições analisadas, a composição asfáltica com grafite cominuído torna-se uma alternativa promissora como material aplicável em pavimentos rodoviários, atuando como substituto do fíler tradicional (cimento Portland).
\end{abstract}

Palavras-chave: Mistura asfáltica; Flexão a quatro pontos; Módulo complexo; Ângulo de fase; Grafite cominuído.

\begin{abstract}
Throughout its history, the construction of road pavements, faces the loss of functional and structural characteristics, due to technological and financial obstacles. In this way, studies of new materials to be used in asphalt concrete become relevant, in order to favor the implementation of more economical maintenance, recovery and pavement design policies due to their greater long-term technical suitability, and in particular, minimize the early maintenance of these structures. In this respect, the main objective of the present work was to experimentally compare through complex module $\left(\mathrm{E}^{*}\right)$ and phase angle $(\delta)$ two types of asphalt concrete, one made with asphalt composition with Portland cement (CA-REF), and the other asphalt composition with comminuted graphite (CA-GRAFC). For this, four point flexion equipment was used, using a uniaxial sinusoidal loading of compression, deformation amplitude of $50 \mu \mathrm{m} / \mathrm{m}$; frequencies of $0.1,0.2,0.5,1,2,5,10$ and $20 \mathrm{~Hz}$ and temperatures from 0 to $40^{\circ} \mathrm{C}$, in increments of $5^{\circ} \mathrm{C}$. After carrying out the mechanical tests, it was found that, under the conditions analyzed, the asphalt composition with comminuted graphite becomes a promising alternative as a material applicable to road pavements, acting as a substitute for the traditional filler (Portland cement).
\end{abstract}

Keywords: Asphalt mixtures; Four point bending test; Complex module; Phase angle; Comminuted graphite. 


\begin{abstract}
Resumen
A lo largo de su historia, la construcción de pavimentos viales, enfrenta la pérdida de características funcionales y estructurales, debido a obstáculos tecnológicos y financieros. De esta manera, adquieren relevancia los estudios de nuevos materiales a ser utilizados en el hormigón asfáltico, con el fin de favorecer la implementación de políticas de mantenimiento, recuperación y diseño de pavimentos más económicas por su mayor idoneidad técnica a largo plazo, y en particular, minimizar el mantenimiento temprano de estas estructuras. En este sentido, el objetivo principal del presente trabajo fue comparar experimentalmente mediante módulo complejo $(\mathrm{E} *)$ y ángulo de fase $(\delta)$ dos tipos de hormigón asfáltico, uno elaborado con composición asfáltica con cemento Portland (CA-REF), y el otro composición asfáltica con grafito triturado (CA-GRAFC). Para ello se utilizó equipo de flexión de cuatro puntos, utilizando una carga de compresión sinusoidal uniaxial, amplitud de deformación de $50 \mu \mathrm{m} / \mathrm{m}$; frecuencias de $0,1,0,2,0,5,1,2,5$, 10 y $20 \mathrm{~Hz}$ y temperaturas de 0 a $40^{\circ} \mathrm{C}$, en incrementos de $5^{\circ} \mathrm{C}$. Tras la realización de los ensayos mecánicos, se constató que, en las condiciones analizadas, la composición asfáltica con grafito triturado se convierte en una alternativa prometedora como material aplicable a pavimentos viales, actuando como sustituto del relleno tradicional (cemento Portland).
\end{abstract}

Palabras clave: Mezcla de asfalto; Flexión de cuatro puntos; Módulo complejo; Ángulo de fase; Grafito triturado.

\title{
1. Introdução
}

Na Geotecnia, uma subárea da Engenharia Civil, os pavimentos são estruturas em multicamadas que visam resistir aos esforços e assegurar uma superfície de rolamento, com segurança e conforto, para o fluxo de veículos e aeronaves (Yoder e Witczak, 1975; Senço, 2007; Balbo, 2007; Bernucci, et al., 2008; Papagiannakis e Massad, 2008; Kim, 2008; Marques e Mendes, 2012; Sol-Sánchez, et al., 2015; Moreno-Navarro e Rubio-Gámez, 2016). Quando se tem pavimentos do tipo flexíveis, estes constituem-se de uma camada superior (revestimento asfáltico), responsável por transmitir as cargas às camadas inferiores (base, sub-base, subleito). Porém, os pavimentos rodoviários ao longo de sua história, percebe-se a ocorrência prematura da perda das características funcionais e estruturais dos pavimentos asfálticos levou ao desenvolvimento e adoção de materiais inovadores para aumentar a vida útil das camadas asfálticas (Moreno-Navarro, Iglesias e Rubio-Gámez, 2016).

À vista disso, com o objetivo de reduzir custos e manutenção e, ainda, mitigar a exploração dos materiais convencionais, recursos naturais não renováveis, normalmente usados nessas estruturas, a literatura cita diversos estudos relacionados a utilização de diferentes materiais, provenientes de processos industriais (Specht, 2012; Bardini, et al., 2012; Yan, Xu e You, 2015; Arao, 2016; Torres, et al., 2019; Pereira, et al., 2019; Carlesso, et al., 2019).

Neste contexto, o presente trabalho apresenta um estudo sobre o desempenho de uma composição asfáltica produzida com grafite cominuído em moinho de alta energia, por meio da determinação do módulo complexo e ângulo de fase, sob flexão a quatro pontos.

\section{Metodologia}

\subsection{Materiais}

\subsubsection{Agregados}

Os materiais utilizados nesta pesquisa foram agregados: brita 1, brita 0 , areia e pó de pedra. As propriedades físicas destes materiais foram obtidas por meio de ensaios preconizados pela American Society for Testing and Materials (ASTM). Para os agregados graúdos (brita 1 e brita 0), realizaram-se ensaios de densidade real (Gsa), densidade aparente (Gsb), absorção de água foram determinadas conforme o método de ensaio C127 (ASTM, 2015) e dureza segundo a norma C131 (ASTM, 2014). Os agregados miúdos (pó de pedra e areia), foram avalizados quanto Gsa, Gsb e absorção de água foram realizados de acordo com as diretrizes propostas pela normaC128 (ASTM, 2015).

Na Tabela 1 são apresentados os resultados dos ensaios realizados, bem como as respectivas normas adotadas. 
Tabela 1: Resultados de testes de rotina para agregados minerais.

\begin{tabular}{|c|c|c|}
\hline Parâmetro & Resultado & Norma \\
\hline Gsa, $\mathrm{g} / \mathrm{cm}^{3}$ (brita 1 ) & 2,58 & \multirow{3}{*}{ ASTM C 127} \\
\hline $\mathrm{Gsb}, \mathrm{g} / \mathrm{cm}^{3}$ (brita 1) & 2,55 & \\
\hline Absorção, \% (brita 1) & 0,51 & \\
\hline Gsa, $\mathrm{g} / \mathrm{cm}^{3}$ (brita 0 ) & 2,74 & \multirow{3}{*}{ ASTM C 127} \\
\hline $\mathrm{Gsb}, \mathrm{g} / \mathrm{cm}^{3}$ (brita 0 ) & 2,62 & \\
\hline Absorção, \% (brita 0) & 1,66 & \\
\hline Gsa, $\mathrm{g} / \mathrm{cm}^{3}$ (areia) & 2,21 & \multirow{2}{*}{ ASTM C 128} \\
\hline Gsa, $\mathrm{g} / \mathrm{cm}^{3}$ (pó de pedra) & 2,75 & \\
\hline
\end{tabular}

Fonte: Autores (2021).

Por meio da citada tabela, pode-se observar que, a absorção das britas 1 e 0 evidenciaram baixos valores (menores que $2 \%$ ), enfatiza-se que uma maior absorção dos agregados graúdos, resulta em um maior consumo de ligante asfáltico. Quanto ao ensaio de abrasão Los Angeles, percebe-se que os agregados, brita 1 e brita 0 , ficaram dentro do limite de $50 \%$ estabelecido pelo Departamento Nacional de Infraestrutura de Transportes (DNIT ME - 035/98).

\subsubsection{Fíler}

Para a composição convencional, teve-se o cimento Portland tipo CP II-E-32 composto por pozolana. Quanto ao grafite cominuído utilizado na presente pesquisa foi obtido por meio do processo de cominuição do grafite comercial, o qual foi adquirido no comércio local do município de Porto Velho, capital do estado de Rondônia.

Tendo-se como motivação que a diminuição do tamanho dos grãos de um material, acarreta, em regra, no aumento da superfície específica do material (aumento do número de átomos neste contorno) que, por conseguinte, potencializa as suas propriedades (Jackson e Sherman, 1953; Suryanarayana, 1995; Das, 2014), buscou-se por meio do moinho de alta energia (MAE), modelo 8000M Mixer/Mill® da SPEX Sample, a diminuição do tamanho médio dos grãos desse mineral. Este método foi utilizado devido sua simplicidade e rápida pulverização das amostras, garantindo também maior homogeneidade para a composição do material.

Nesse aspecto, estudou-se a sua redução por 4 horas. O processo de moagem teve início com a pesagem da amostra, e na sequência inseriu-se o material, 4 gramas no recipiente cilíndrico ( $D=57,15 \mathrm{~mm}$ e $\mathrm{h}=76,20 \mathrm{~mm}$ ) com capacidade de carga de até 10 gramas, juntamente com duas esferas de aço de 12,70mm para, então, dar início ao processo de cominuição mecânica.

\subsection{Cimento asfáltico de petróleo}

Como aglomerante orgânico foi utilizado o cimento asfáltico de petróleo (CAP), fornecido por uma empresa do município de Manaus, capital do estado do Amazonas. O material foi classificado quanto à penetração como 50/70, cujas especificações estão de acordo com a agência Nacional de Petróleo (ANP), que utiliza os métodos do órgão estadunidense de normalização da ASTM, cujos os parâmetros de aceitação e classificação são apresentados na Tabela 2. 
Tabela 2: Propriedades do ligante asfáltico.

\begin{tabular}{|c|c|c|}
\hline Propriedades & Resultados & Métodos \\
\hline Penetração $-5 \mathrm{~s}, 25^{\circ} \mathrm{C}$ & $67 \mathrm{~mm}$ & ASTM D5 \\
\hline Ponto de Amolecimento, min & $50,1^{\circ} \mathrm{C}$ & ASTM D36 \\
\hline Viscosidade Saybolt Furol a $135^{\circ} \mathrm{C}$ & $284 \mathrm{~s}$ & \multirow{3}{*}{ ASTM E102 } \\
\hline Viscosidade Saybolt Furol a $150^{\circ} \mathrm{C}$ & $142,7 \mathrm{~s}$ & \\
\hline Viscosidade Saybolt Furol a $177^{\circ} \mathrm{C}$ & $48,6 \mathrm{~s}$ & \\
\hline Viscosidade Brookfield a $135^{\circ} \mathrm{C}$ & $537 \mathrm{cP}$ & \multirow{3}{*}{ ASTM D4402 } \\
\hline Viscosidade Brookfield a $150^{\circ} \mathrm{C}$ & $277,8 \mathrm{cP}$ & \\
\hline Viscosidade Brookfield a $177^{\circ} \mathrm{C}$ & $95,8 \mathrm{cP}$ & \\
\hline Ponto de fulgor, min & $317^{\circ} \mathrm{C}$ & ASTM D92 \\
\hline Solubilidade em tricloroetileno & $99,5 \%$ & ASTM D2042 \\
\hline RTFOT Variação em massa & $0,04 \%$ & ASTM D2872 \\
\hline Ductilidade a $25^{\circ} \mathrm{C}$ & $>100 \mathrm{~cm}$ & ASTM D113 \\
\hline RTFOT aumento do ponto de amolecimento & $7,1^{\circ} \mathrm{C}$ & ASTM D36 \\
\hline RTFOT penetração retida & $64 \%$ & ASTM D5 \\
\hline
\end{tabular}

Fonte: Autores (2021).

\subsection{Dosagem asfáltica}

Em relação as dosagens asfálticas, buscou-se o enquadramento dos materiais nos limites estabelecidos para a Faixa C da norma ES031 (DNIT, 2004). Após a determinação da composição da mistura de referência contendo apenas materiais minerais, a composição granulométrica dos agregados foi realizada utilizando-se o método tradicional de tentativas.

A partir da mistura de referência (CA-REF), foram utilizadas as metodologias do método Bailey para proporcionamento dos componentes, detalhado por Mendes e Marques (2012) obtendo-se, assim, uma composição que se enquadrasse nos parâmetros do citado método, bem como nos limites da Faixa C do DNIT. O mesmo procedimento foi utilizado para a mistura com grafite cominuído (CA-GRAFC).

\subsection{Procedimento experimental das misturas asfálticas}

As composições estudadas foram submetidas ao ensaio de flexão a quatro pontos, conforme as recomendações da norma europeia 12697-26 (EN, 2018), com aplicação de carregamento senoidal e amplitude de deformação de 50 $\mu$ m/m. A série de frequências de aplicação de carga foram os valores de $0,1,0,2,0,5,1,2,5,10,20 \mathrm{~Hz}$; e temperaturas de 0 até $40^{\circ} \mathrm{C}$, com incrementos de 5 em $5^{\circ} \mathrm{C}$.

\section{Resultados e Discussão}

\subsection{Parâmetros mecânicos}

As Tabelas 3 e 4 mostram uma visão geral dos resultados obtidos de módulo complexo correspondentes às médias aritméticas obtidas para três corpos de prova. De modo geral, percebe-se o aumento dos módulos complexos para a mistura CA-GRAFC respeitante à mistura CA-REF, o que significa que o material cominuído aumenta a rigidez das misturas asfálticas. 
Tabela 3: Módulo complexo, CA-REF.

\begin{tabular}{c|c|c|c|c|c|c|c}
\hline & $\mathbf{0}^{\circ} \mathbf{C}$ & $\mathbf{5}^{\circ} \mathbf{C}$ & $\mathbf{1 0}^{\circ} \mathbf{C}$ & $\mathbf{1 5}^{\circ} \mathbf{C}$ & $\mathbf{2 0}^{\circ} \mathbf{C}$ & $\mathbf{2 5}^{\circ} \mathbf{C}$ & $\mathbf{3 0}^{\circ} \mathbf{C}$ \\
\hline $\mathbf{0 , 1 ~ H z}$ & 3806 & 1724 & 1433 & 1046 & 450 & 390 & 256 \\
\hline $\mathbf{0 , 2} \mathbf{~ H z}$ & 4046 & 2106 & 1712 & 1219 & 557 & 464 & 286 \\
\hline $\mathbf{0 , 5} \mathbf{~ H z}$ & 4464 & 2538 & 2125 & 1536 & 744 & 606 & 362 \\
\hline $\mathbf{1 ~ H z}$ & 4854 & 2969 & 2473 & 1794 & 914 & 754 & 436 \\
\hline $\mathbf{2 ~ H z}$ & 4972 & 3366 & 2846 & 2153 & 1131 & 945 & 532 \\
\hline $\mathbf{5 ~ H z}$ & 5184 & 3872 & 3381 & 2565 & 1502 & 1188 & 697 \\
\hline $\mathbf{1 0 ~ H z}$ & 5214 & 4294 & 3748 & 2911 & 1780 & 1370 & 840 \\
\hline $\mathbf{2 0 ~ H z}$ & 5360 & 4552 & 4284 & 3339 & 2316 & 1645 & 992 \\
\hline
\end{tabular}

Fonte: Autores (2021).

Tabela 4: Módulo complexo, CA-GRAFC.

\begin{tabular}{c|c|c|c|c|c|c|c}
\hline & $\mathbf{0}^{\circ} \mathbf{C}$ & $\mathbf{5}^{\circ} \mathbf{C}$ & $\mathbf{1 0}^{\circ} \mathbf{C}$ & $\mathbf{1 5}^{\circ} \mathbf{C}$ & $\mathbf{2 0}^{\circ} \mathbf{C}$ & $\mathbf{2 5}^{\circ} \mathbf{C}$ & $\mathbf{3 0}^{\circ} \mathbf{C}$ \\
\hline $\mathbf{0 , 1 ~ H z}$ & 4707 & 2791 & 1967 & 1552 & 911 & 589 & 413 \\
\hline $\mathbf{0 , 2} \mathbf{~ H z}$ & 4852 & 3395 & 2184 & 1873 & 1029 & 692 & 476 \\
\hline $\mathbf{0 , 5} \mathbf{~ H z}$ & 5511 & 3979 & 2567 & 2249 & 1230 & 869 & 594 \\
\hline $\mathbf{1 ~ H z}$ & 5860 & 4390 & 2894 & 2585 & 1362 & 1022 & 704 \\
\hline $\mathbf{2 ~ H z}$ & 6207 & 4745 & 3174 & 2976 & 1586 & 1218 & 846 \\
\hline $\mathbf{5 ~ H z}$ & 6345 & 4899 & 3467 & 3417 & 1940 & 1558 & 1143 \\
\hline $\mathbf{1 0 ~ H z}$ & 6578 & 5014 & 3675 & 3584 & 2193 & 1770 & 1326 \\
\hline $\mathbf{2 0 ~ H z}$ & 6804 & 5394 & 3930 & 3706 & 2510 & 2193 & 1526 \\
\hline
\end{tabular}

Fonte: Autores (2021).

Para evidenciar melhor os aumentos no módulo complexo ocasionado pela incorporação do grafite cominuído nas misturas asfálticas, assim como obter uma descrição completa do desempenho mecânico das composições em estudo, utilizouse o princípio da superposição frequência-temperatura para a construção das curvas mestras. A importância destas curvas reside em poder definir graficamente o módulo de rigidez para diversas temperaturas e frequências, muito além das utilizados nos testes laboratoriais, o qual pode-se ampliar significativamente a margens de dados.

No caso específico, embora os ensaios realizados tenham se limitado a frequências compreendidas entre $0,1 \mathrm{a} 20 \mathrm{~Hz}$, com a construção das curvas mestras, consegue-se determinar o citado parâmetro para frequências entre 0,0001 e $100000000 \mathrm{~Hz}$. Ou seja, é possível a partir do princípio da superposição frequência-temperatura obter valores de módulo complexo para frequências extremamente altas e baixas. Na prática, seria impraticável, pois exigiria longos períodos de investigação para as frequências extremamente baixas, e intervalos de tempo ao nível de vibração molecular nas frequências altas (MELO, 2014).

Com as frequências de ensaio 0,1 a $20 \mathrm{~Hz}$ e temperaturas utilizadas 0 a $30^{\circ} \mathrm{C}$, foram calculados os fatores de translação horizontal (aT) por meio da Equação 1 de WilliamsLandel-Ferry (WLF). Na Figura 1, são apresentadas as curvas mestras das misturas CA-REF e CA-GRAFC.

$$
\log a_{T}=\frac{-C_{1} x\left(T-T_{r e f}\right)}{C_{2}+\left(T-T_{r e f}\right)}
$$




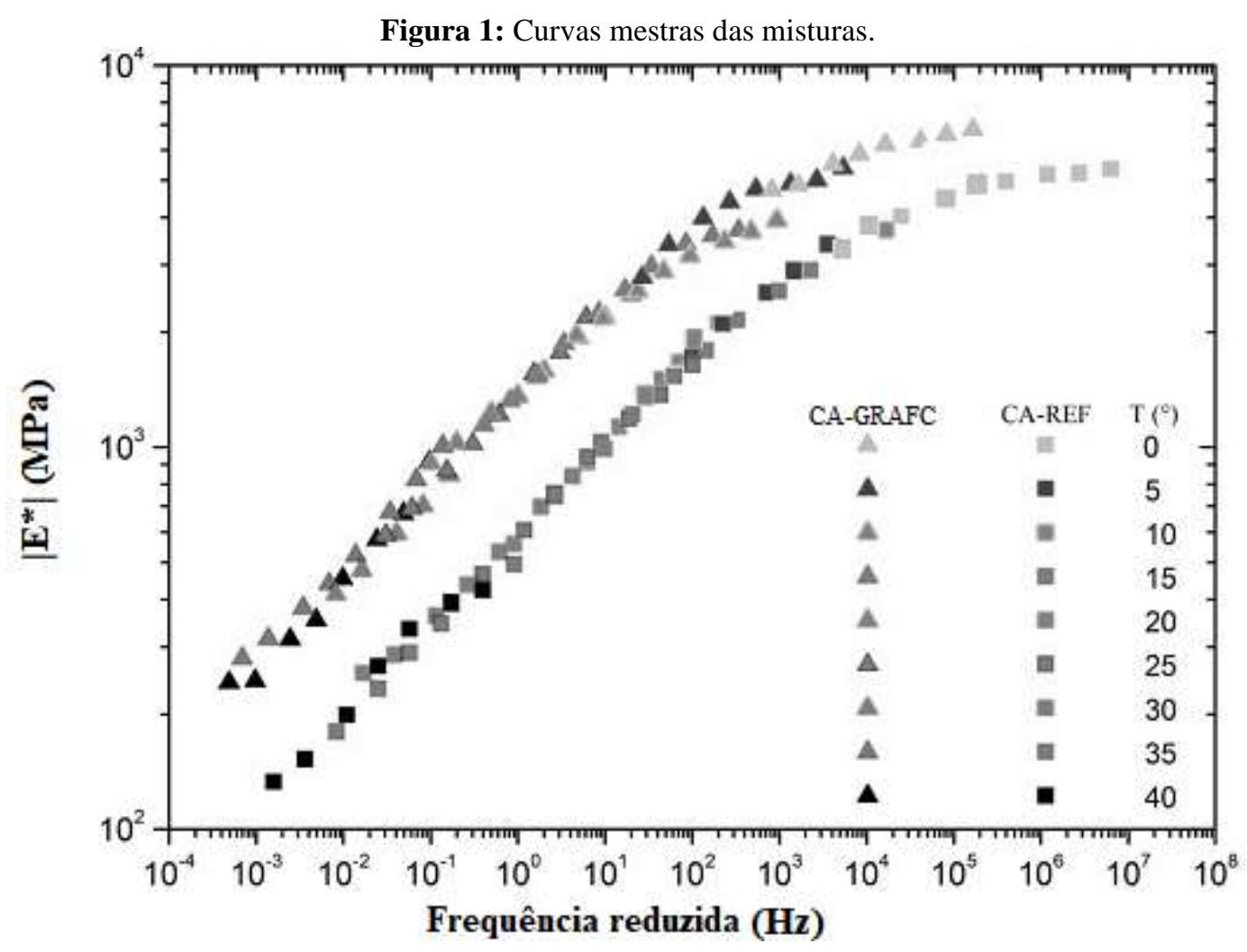

Fonte: Autores (2021).

Na Figura 1, observa-se que a mistura CA-GRAFC apresentou melhores resultados de rigidez, em todo o domínio de frequências, quando comparado com a composição com CA-REF, semelhante ao reportado por Melo e Trichês (2016) e Carlesso et. al. (2019). Em termos mecânicos, os autores observaram que a incorporação de materiais alternativos na mistura asfáltica, resulta em módulos e ângulos de fases superiores em comparação a mistura convencional.

Todavia, vale ressaltar que os concretos asfálticos avaliados no presente estudo não se comportaram eficientemente como os trabalhos pesquisada na literatura. $\mathrm{O}$ motivo pelo qual que as misturas testadas no presente estudo não se comportaram eficientemente como os trabalhos pesquisada na literatura pode ser atribuído ao processo de compactação dos corpos prismáticos. No presente estudo foi empregado o sistema de compactação usado por Mello (2008), Lima (2017) e Torres (2018). Enquanto os autores Melo (2014), Melo e Trichês (2016), Marcon (2016), Carlesso (2017) e Carlesso et. al. (2019) utilizaram a mesa compactadora LCPC desenvolvida na França pelo Laboratoire Central des Ponts et Chaussées. Além das diferenças metodológicas, outro fator que possivelmente gera esta variação e dispersão dos resultados nos parâmetros de resistência, pode ser as diferentes dosagens asfálticas adotadas pelos autores, e sugere-se que esses fatores também possam ter contribuído para essa diferença.

A Tabela 5 apresenta, em termos percentuais, o aumento da rigidez, em que explicita os ganhos do módulo complexo da formulação asfáltica CA-GRAFC relativa à mistura asfáltica CA-REF. 
Tabela 5: Aumentos no módulo complexo da mistura asfáltica CA-GRAFC em relação a mistura asfáltica CA-REF (ganhos em porcentagem).

\begin{tabular}{c|c|c|c|c|c|c|c}
\hline \multicolumn{8}{c}{ Termos percentuais (\%) } \\
\hline & $\mathbf{0}{ }^{\circ} \mathbf{C}$ & $\mathbf{5}^{\circ} \mathbf{C}$ & $\mathbf{1 0}{ }^{\circ} \mathbf{C}$ & $\mathbf{1 5}^{\circ} \mathbf{C}$ & $\mathbf{2 0}^{\circ} \mathbf{C}$ & $\mathbf{2 5}^{\circ} \mathbf{C}$ & $\mathbf{3 0}^{\circ} \mathbf{C}$ \\
\hline $\mathbf{0 , 1 ~ H z}$ & 23,65 & 61,89 & 37,26 & 48,34 & 102,44 & 50,97 & 61,01 \\
\hline $\mathbf{0 , 2} \mathbf{~ H z}$ & 19,92 & 61,21 & 27,56 & 53,68 & 84,74 & 48,90 & 66,32 \\
\hline $\mathbf{0 , 5 ~ H z}$ & 23,44 & 56,76 & 20,80 & 46,41 & 65,32 & 43,35 & 64,27 \\
\hline $\mathbf{1 ~ H z}$ & 20,73 & 47,88 & 17,03 & 44,11 & 49,02 & 35,55 & 61,62 \\
\hline $\mathbf{2 ~ H z}$ & 24,84 & 40,99 & 11,52 & 38,22 & 40,23 & 28,83 & 58,96 \\
\hline $\mathbf{5 ~ H z}$ & 22,40 & 26,52 & 2,55 & 33,21 & 29,16 & 31,10 & 64,04 \\
\hline $\mathbf{1 0 ~ H z}$ & 26,16 & 16,76 & 1,95 & 23,11 & 23,20 & 29,23 & 57,84 \\
\hline $\mathbf{2 0 ~ H z}$ & 26,93 & 18,51 & 5,91 & 10,98 & 8,38 & 33,35 & 53,83 \\
\hline
\end{tabular}

Fonte: Autores (2021).

Consolidando-se os valores dos ganhos em porcentagem de rigidez para melhor visualização dos resultados, têm-se os gráficos dispostos na Figura 2 que demonstra os aumentos da adição do CA-GRAFC em comparação a mistura de referência. Consoante aos resultados para a composição CA-GRAFC, e tomando como exemplo as frequências de 1 e 10Hz, representativas de uma velocidade, na prática, de 20 e $72 \mathrm{~km} / \mathrm{h}$. Para frequência de $1 \mathrm{~Hz}$ a adição do módulo complexo é da ordem de $61,62,35,55,49,02$ e 44,11\%, para as temperaturas de $30,25,20$ e $15^{\circ} \mathrm{C}$, respectivamente. No caso da frequência de $10 \mathrm{~Hz}$, o acréscimo é na ordem de 57,84, 29,23, 23,20 e 23,11\% nas aludidas temperaturas.

Nota-se que, existe diversas variações de porcentagens ao longo do gráfico, todavia em todos os pontos de estudo houveram acréscimo de rigidez. Além disso, enfatiza-se que, na prática, o aumento da rigidez, retrata um maior coeficiente angular na curva tensão-deformação (Melo, 2014). Isso indica que a rigidez da mistura CA-GRAFC, em campo, sob um mesmo estado de tensões, seriam menos sensíveis às deformações de tração na fibra inferior da camada de revestimento asfáltico quando comparado à mistura CA-REF. Ou seja, a utilização do grafite cominuído é favorável ao aumento de rigidez.

Figura 2: Ganhos no módulo complexo em porcentagens.

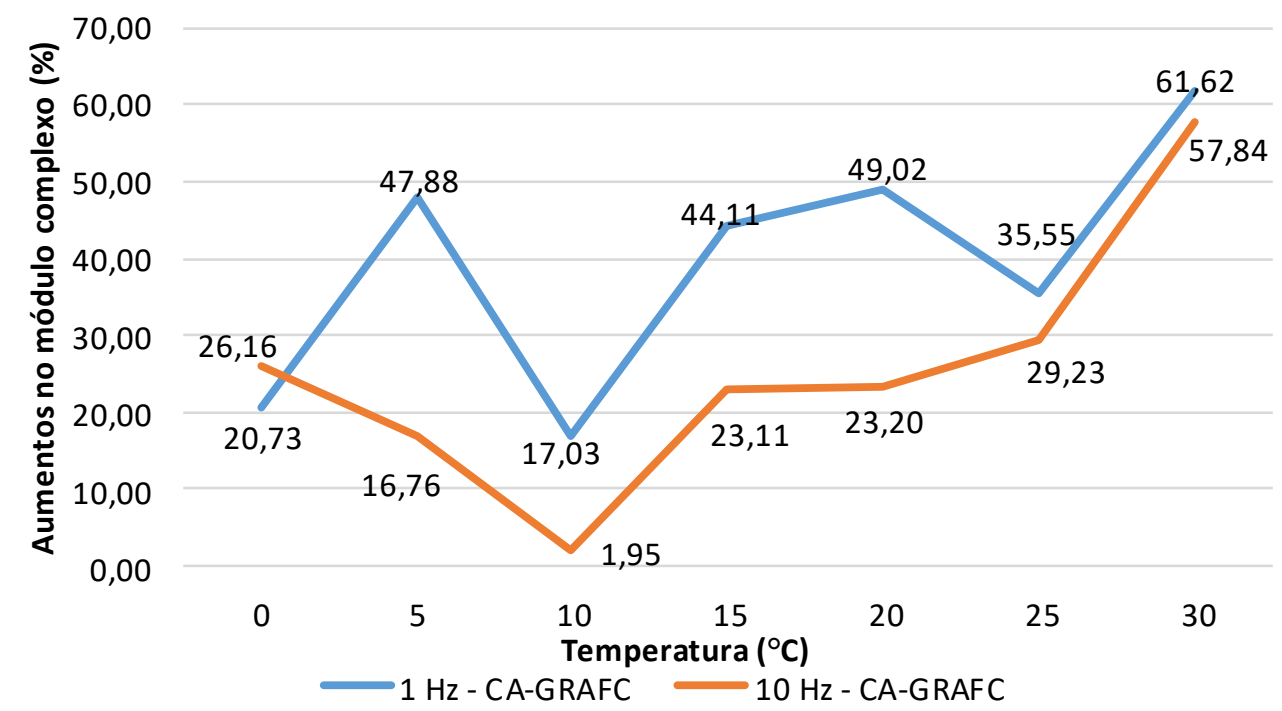

Fonte: Autores (2021).

Na Figura 3 têm-se o modulo de rigidez dinâmico em função da temperatura. Estas curvas correlacionam o módulo complexo (escala logarítmica) com a temperatura (escala aritmética), e expressam a variação da rigidez em função das 
diferentes temperaturas e frequências de carregamento. Deste modo, é possível analisar o comportamento térmico das misturas asfálticas a partir das inclinações das curvas, isto é, quanto maior a temperatura menor é a rigidez do concreto asfáltico.

É possível observar que as isócronas apresentam a redução da rigidez a medida que há um aumento da temperatura de ensaio, ou seja, o valor de módulo complexo é inversamente proporcional ao aumento de temperatura. Tais observações já eram esperadas, uma vez que para materiais viscoelásticos, o aumento de temperatura diminui sua rigidez.

Constata-se, também, um melhor comportamento térmico para as misturas CA-GRAFC no tocante à formulação referência. Em suma, isto indicaria que, em campo, as mudanças de temperatura exerceriam menor influência na rigidez da mistura com grafite cominuído.

Figura 3: Isócronas, mistura CA-REF x mistura CA-GRAFC.

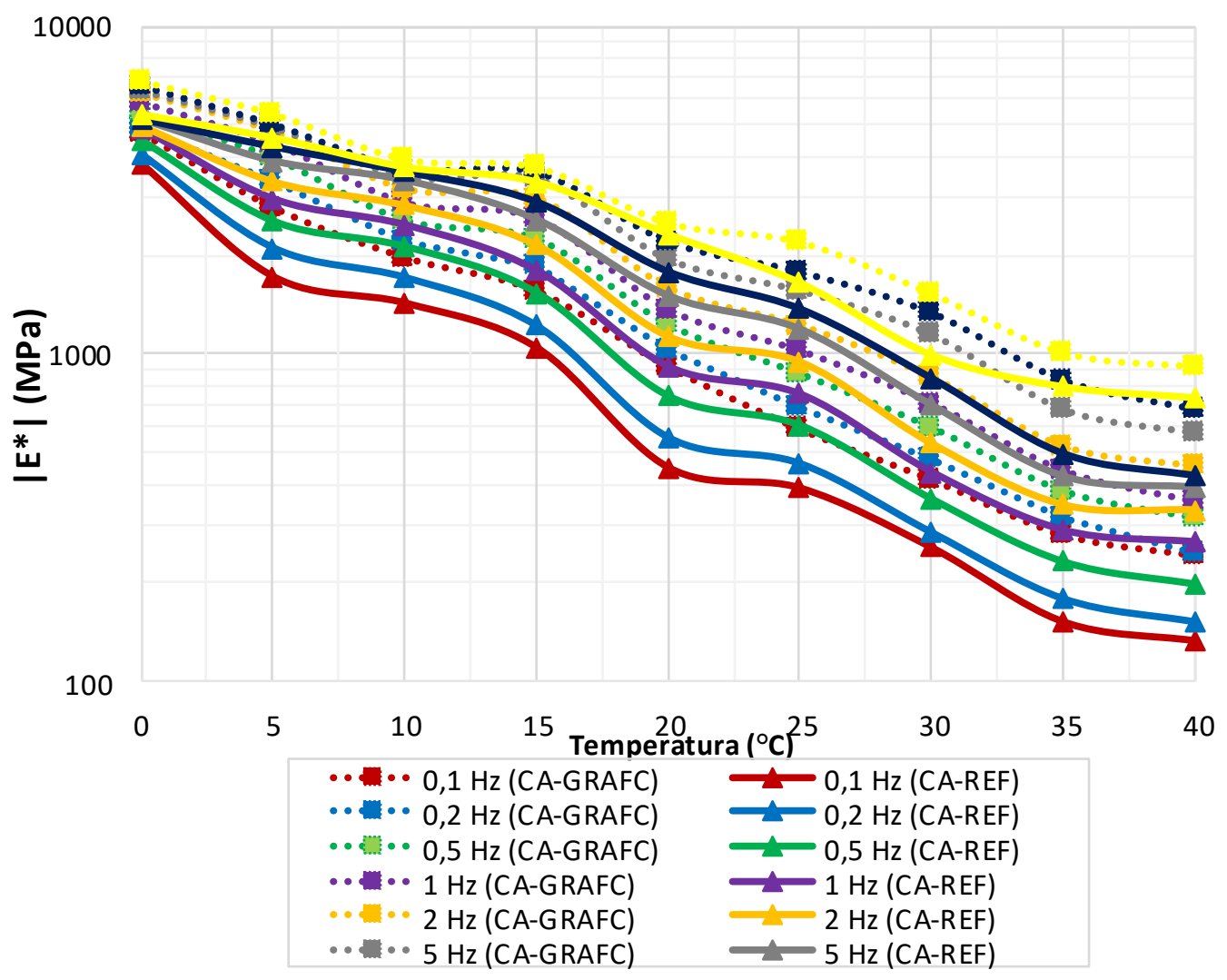

Fonte: Autores (2021).

Em relação ao ângulo de fase, apresenta-se na Figura 4 o diagrama de Black para as misturas CA-REF e CA-GRAFC, que relaciona o módulo complexo (escala logarítmica) com os respectivos valores de ângulo de fase (escala aritmética), em função da frequência de carregamento e temperatura de ensaios.

De acordo com a mencionada figura, constata-se um encurtamento do espaço de Black da mistura CA-GRAFC em comparação a CA-REF. Em adição, observa-se uma tendência de aumento dos ângulos de fase, à medida em que se aumenta a temperatura de teste. Portanto, a incorporação do material grafite cominuído, em linhas gerais, resulta na redução dos ângulos de fase, tornando as misturas asfálticas mais elásticas. 
Figura 4: Espaço de Black, mistura CA-REF x mistura CA-GRAFC.

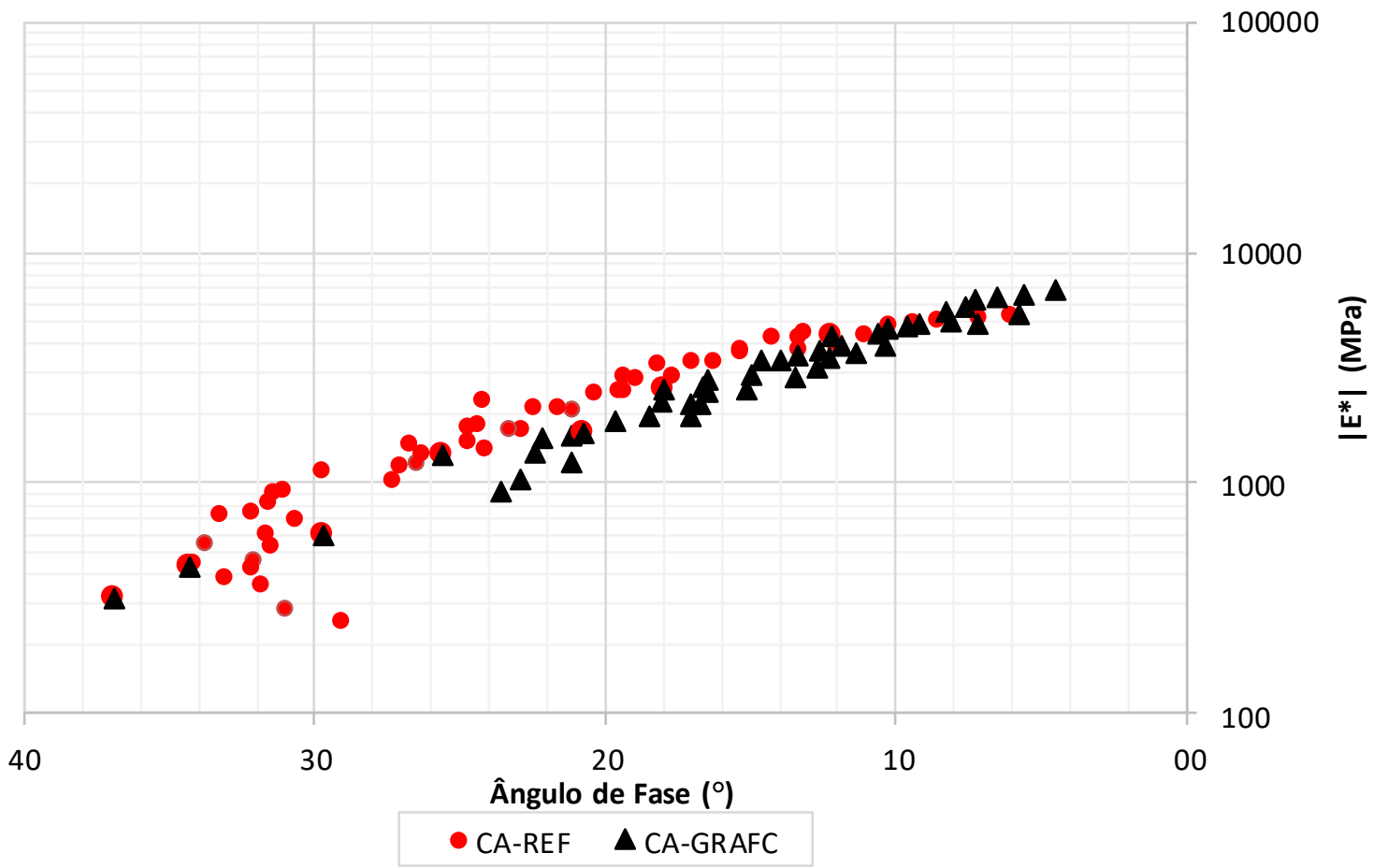

Fonte: Autores (2021).

\section{Considerações Finais}

Este trabalho teve como objetivo analisar, por meio de ensaios laboratoriais, o comportamento mecânico de concretos asfálticos produzidos com grafite cominuído.

Por meio dos resultados encontrados, constatou-se que a substituição do fíler tradicional por grafite cominuído promoveu um aumento de rigidez, ou seja, o concreto asfáltico CA-GRAFC apresentou resultados superiores de módulo complexo quando comparado aos resultados do concreto betuminoso convencional, na prática, a mistura CA-GRAFC seria mais resistência à deformação permanente do que a mistura CA-REF.

De forma geral, concluiu-se que a utilização do grafite cominuído, como fíler, proporciona melhoria tanto no quesito de desempenho mecânico de concretos asfálticos, quanto para o meio ambiente, o qual favorece a implementação de políticas de manutenção, recuperação e projetos de pavimentos rodoviários mais econômicos pela sua maior adequabilidade técnica a longo prazo, e, em especial, minimiza a exploração dos materiais não renováveis normalmente usados nessas estruturas.

\section{Referências}

ASTM C127-15. (2015). Standard test method for density, relative density (specific gravity), and absorption of coarse aggregate. Conshohocken: ASTM International. 10.1520/C0127-15.

ASTM C128-15. (2015). Standard Test Method for Relative Density (Specific Gravity) and Absorption of Fine Aggregate. Conshohocken: ASTM International. 10.1520/C0128-15.

ASTM C131/C131M-14. (2014). Standard Test Method for Resistance to Degradation of Small-Size Coarse Aggregate by Abrasion and Impact in the Los Angeles Machine. Conshohocken: ASTM International. 10.1520/C0131_C0131M-14.

ASTM D5/D5M-20. (2013). Standard test method for penetration of bituminous materials. Conshohocken: ASTM International. 10.1520/D0005_D0005M-20.

ASTM D36/D36M-14. (2014). Standard test method for softening point of bitumen (ring-and-ball apparatus). Conshohocken: ASTM International. 10.1520/D0036_D0036M-14R20. 
ASTM D92-18. (2018). Standard Test Method for Flash and Fire Points by Cleveland Open Cup Tester. Conshohocken: ASTM International. 10.1520/D009218.

ASTM D113-17. (2017). Standard Test Method for Ductility of Asphalt Materials. Conshohocken: ASTM International. 10.1520/D0113-17.

ASTM D2042-15. (2015). Standard Test Method for Solubility of Asphalt Materials in Trichloroethylene. Conshohocken: ASTM International. $10.1520 / \mathrm{D} 2042-15$.

ASTM D2872-19. (2012). Standard Test Method for Effect of Heat and Air on a Moving Film of Asphalt (Rolling Thin-Film Oven Test). Conshohocken: ASTM International. 10.1520/D2872-19.

ASTM D4402/D4402M-15. (2013). Standard test method for viscosity determination of asphalt at elevated temperatures using a rotational viscometer. Conshohocken: ASTM International. 10.1520/D4402_D4402M-15.

ASTM E102/E102M-93. (2016). Standard Test Method for Saybolt Furol Viscosity of Bituminous Materials at High Temperatures. Conshohocken: ASTM International. 10.1520/E0102_E0102M-93R16.

Arao, M. (2016). Avaliação do comportamento mecânico de misturas asfálticas com a inserção de polietileno tereftalato (PET) triturado. Pontifícia Universidade Católica do Rio de Janeiro, Dissertação de Mestrado.

Balbo, J. T. (2007). Pavimentação asfáltica: materiais, projeto e restauração. Editora Oficina de Textos, 560 p.

Bardini, V. S. S., Klinsky, L. M. G., Júnior, J. L. F. \& Roque, R. (2012). Influência do fíler mineral no comportamento reológico de mástiques asfálticos. Transportes, 20 (3), 19-26. https://doi.org/10.4237/transportes.v20i4.603.

Bernucci, L. B., Motta, L. M. G., Ceratti, J. A. P. \& Soares, J. B. (2008). Pavimentação asfáltica: formação básica para engenheiros. 501 p.

Carlesso, G. C. (2017). Estudo do comportamento de mistura asfáltica modificada por nanoargila e polímero SBS. Universidade Federal de Santa Catarina, Dissertação de mestrado.

Carlesso, G. C., Trichês, G., De Melo, J. V.S., Marcon, M. F., Thives, L. P. \& Da Luz, L. C. (2019). Evaluation of Rheological Behavior, Resistance to Permanent Deformation, and Resistance to Fatigue of Asphalt Mixtures Modified with Nanoclay and SBS Polymer. Applied Sciences-Basel, 9, 2697-2713. https://doi.org/10.3390/app9132697

Das, B.M. (2015). Fundamentos de engenharia geotécnica. Cengage Learning. 612 p.

DNIT ES 031/2006. (2006). Pavimentos flexíveis: concreto asfáltico: especificação de serviço.

DNIT ME 035/1998. (1998). Agregados - determinação da abrasão "Los Angeles".

EN 12697-26. (2018). Bituminous mixtures - test methods for hot mix asphalt, part 26: Stiffness.

Jackson, M. L. \& Sherman, G. D. (1953). Chemical weathering of minerals in soil. Adv. Agron, 211-318.

Kim, Y. R. (2008). Modeling of asphalt concrete. United State of America: ASCE Press. 460 p.

Marcon, M. F. (2016). Estudo e comparação do desempenho mecânico e reológico entre concretos asfálticos modificados por polímero SBS, borracha moída de pneu e nanomateriais. Universidade Federal de Santa Catarina. Dissertação de Mestrado.

Mendes, L. O. \& Marques, G. L. O. (2012). Avaliação da influência do método bailey no processo de dosagem e desempenho de misturas asfálticas. Transportes, 20 (4), 35-43. 10.4237/transportes.v20i4.574.

Melo, J. V. S. (2014). Desenvolvimento e estudo do comportamento reológico e desempenho mecânico de concretos asfálticos modificados com nanocompósitos. Universidade Federal de Santa Catarina, Tese de Doutorado.

Melo, J. V. S. \& Trichês, G. (2016). Effects of organophilic nanoclay on the rheological behavior and performance leading to permanent deformation of asphalt mixtures. Journal of Materials in Civil Engineering, 28 (11), 04016142. 10.1061/(ASCE)MT.1943-5533.0001650.

Moreno-Navarro, F. \& Rubio-Gámez, M. C. (2016). A review of fatigue damage in bituminous mixtures: Understanding the phenomenon from a new perspective. Construction and Building Materials, 113, 927-938. https://doi.org/10.1016/j.conbuildmat.2016.03.126.

Papagiannakis, A. T. \& Massad, E. A. (2008). Pavement Design and Materials. John Wiley \& Sons, Hoboken, 542 p.

Pereira, A. G., Spinola, J. R., Monteiro, A. K. C., Lacerda, B. M., Gusmao, F. S. \& Frota, C. A. (2019). Mechanical behavior of asphaltic mixtures produced with spray graphite in high energy mill. International Journal of Engineering Research and Applications (IJERA), 9, 18-26. 10.9790/9622- 0911031826.

Senço, W. (2007). Manual de técnicas de pavimentação. (2a ed.), Pini, 764 p.

Sol-Sánchez, M., Moreno-Navarro, F., García-Travé, G. \& Rubio-Gámez, M. C. (2015). Laboratory study of the long-term climatic deterioration of asphalt mixtures. Construction and Building Materials, 88, 32-40. https://doi.org/10.1016/j.conbuildmat.2015.03.090.

Specht, L. P., Granich, A., Pasche, E. \& Boeira, F. (2012). Estudo laboratorial de misturas asfálticas com adição de diferentes teores e tipos de cal. Revista Estradas, 17, 66-72.

Suryanarayana, C. (1995). Nanocrystalline materials. Int. Materials Reviews, 40 (20), 41-64. 
Research, Society and Development, v. 10, n. 2, e25110212030, 2021

(CC BY 4.0) | ISSN 2525-3409 | DOI: http://dx.doi.org/10.33448/rsd-v10i2.12030

Torres, A. P., Pereira, I. N. A., Spinola, J. R., Pereira, A. G. \& Frota, C. A. (2019). Four-point bending mechanical behavior of aged asphalt mixtures containing charcoal. International Journal for Innovation Education and Research, 7, 460-472. 10.31686/ijier.Vol7.Iss 10.1794.

Yan, K., Xu, H. \& You, L. (2015). Rheological properties of asphalts modified by waste tire rubber and reclaimed low density polyethylene. Construction and Building Materials, 83, 143-149. https://doi.org/10.1016/j.conbuildmat.2015.02.092.

Yoder, E. J., \& Witczak, M. W. (1975). Principles of Pavement Design. (2a ed.), John Wiley \& Sons Inc. 